\title{
Factores de riesgos psicosociales y estrés percibido en docentes universitarios
}

\section{Psychosocial Risk Factors and Perceived Stress in University Professors}

\author{
Daniel Rubén Tacca Huamán* \\ Universidad Tecnológica del Perú, Lima, Perú \\ ORCID: http://orcid.org/0000-0002-0694-5262 \\ Ana Luisa Tacca Huamán \\ Asociación Educativa Estándares, Lima, Perú \\ ORCID: http://orcid.org/0000-0003-2068-7527
}

Recibido 31-03-19 Revisado 01-07-19 Aprobado 10-09-19 En línea 11-09-19

*Correspondencia

Email: c17500@utp.edu.pe

\section{Citar como:}

Tacca Huamán, D., \& Tacca Huamán, A. (2019). Factores de riesgos psicosociales y estrés percibido en docentes universitarios. Propósitos y Representaciones, 7(3), 323353. doi: http://dx.doi.org/10.20511/pyr2019.v7n3.304 


\section{Resumen}

Los docentes universitarios están involucrados en un ámbito laboral donde interactúan con personas (jefes, colegas, estudiantes, etc.) y situaciones que pueden causar estrés. La presente investigación tuvo como objetivo central conocer la relación entre los factores de riesgos psicosociales y el estrés percibido por los docentes universitarios. El enfoque del trabajo fue cuantitativo, el diseño fue correlacional y transversal; la muestra estuvo constituida por 117 docentes universitarios de Lima, Perú. Los resultados indicaron que el estrés percibido se relaciona positivamente con las exigencias psicológicas, trabajo activo, compensaciones y doble presencia del docente; sin embargo, se encontró una relación inversa entre el estrés y el apoyo social recibido. Los datos muestran que los docentes contratados presentan mayor nivel de estrés y que los principales síntomas experimentados son el cansancio, no poder dormir, dolores de cabeza, poca concentración y variación del apetito. No se encontraron diferencias relevantes en cuanto a la edad y sexo.

Palabras claves: Estrés; Docentes; Psicología; Educación.

\section{Summary}

University teachers are involved in a work environment where they interact with people (bosses, colleagues, students, etc.) and situations that can cause stress. The main objective of this research was to know the relationship between psychosocial risk factors and the stress perceived by university teachers. The focus of the work was quantitative, the design was correlational and transversal; the sample consisted of 117 university teachers from Lima, Peru. The results indicated that perceived stress is positively related to psychological demands, active work, compensations and double presence of the teachers; however, an inverse relationship was found between stress and social support received. The data showed that teachers who work by contract have a higher level of stress and that the main symptoms experienced are tiredness, not being able to sleep, headaches, poor concentration and variation in appetite. No relevant differences were found in terms of age and sex.

Keywords: Stress; Professors; Psychology; Education.

\section{Introducción}

Desde la década de 1980 el mundo laboral ha sufrido grandes cambios que afectan directamente la relación persona-trabajo (Güilgüiruca, Meza, Góngora \& Moya, 2015). Estos cambios son producto del avance en la tecnología, la competencia a nivel mundial, la búsqueda del desarrollo, los mercados y la reducción del Estado; además, influyen en las opciones de empleo, relaciones sociales, tipos de formación, actualización, desarrollo de habilidades, ritmos de trabajo y en la salud de los colaboradores (Gil-Monte, 2012). Todo contexto laboral trae consigo factores de riesgo potenciales para la salud, estas situaciones se configuran según la actividad desarrollada o el ambiente en el cual se desempeña el trabajador (Rodríguez, 2009).

Moreno (2011) explica que, históricamente, se ha prestado atención a los riesgos relacionados con agentes químicos, físicos y ambientales. Sin embargo, Díaz (2009) considera importante los riesgos derivados de la seguridad, infraestructura, naturaleza del proceso productivo, organización de la institución, proceso de adaptación al trabajo y del factor humano. Para Caballero-Lozada y Nieto (2015), el trabajo es una actividad importante en la vida del ser humana y recomiendan realizar un análisis de su concepción teórica y práctica si se desea promover el bienestar laboral y prevenir los riesgos profesionales y psicosociales. Los factores de riesgo psicosociales asociados al trabajo se identifican como ciertas condiciones del ámbito laboral relacionados con la organización, el puesto, la actividad laboral y el entorno (Gil-Monte, 2012), que pueden afectar gravemente la salud física, mental y social de los trabajadores (Moreno, 
2011); el estrés y la violencia laboral son los efectos negativos más comunes (Raffo, Ráez \& Cachay, 2013).

Esteve (citado en Pérez, 2012) señala que en la profesión docente se puede identificar dos grandes aspectos: (1) la vocación por servir a la sociedad, que se relaciona con la realización y satisfacción personal, y (2) los aspectos negativos que logran desequilibrar la salud física y emocional de los docentes. El ámbito laboral del docente universitario es complejo, dinámico y demanda el uso de recursos cognitivos, físicos, emocionales y axiológicos que no se exigen en otros campos profesionales; esto sumado a los cambios promovidos por la globalización, la adopción de nuevos paradigmas educativos y el rol protagónico del estudiante en el proceso de aprendizaje, configuran un escenario multidimensional donde el docente convive día a día con situaciones de riesgo que pueden afectar su salud física y psicológica. Estas coyunturas ocupacionales pueden generar tensión en los docentes debido al desequilibrio entre las exigencias del trabajo y las habilidades que posee para manejarlas (Carlotto \& Gonçalves, 2017).

Cuando se habla de estrés, comúnmente, se refiere a la tensión nerviosa, al desequilibrio emocional o al agente que causa dicha tensión (Ayuso, 2006); Peiró y Rodríguez (2008) consideran que el estrés es un desajuste subjetivo de la persona que se produce al percibir demandas o amenazas que no se pueden controlar y que ocasionan consecuencias negativas. Lazarus y Folkman (1986) consideran al estrés como un proceso dinámico e interactivo que se origina a partir de la relación peculiar y única entre la persona y un ambiente desafiante en comparación con los recursos disponibles; si la persona percibe que esta situación amenazante sobrepasa sus defensas, experimenta estrés (Pieró, 2001). En el modelo de apreciación cognitiva propuesto por Lazarus y Folkman (1986), se distingue la apreciación primaria y secundaria. Durante la apreciación primaria, la persona identifica si un evento es irrelevante, benigno o negativo; la apreciación secundaria se produce cuando, una vez que el evento se catalogó como negativo, la persona evalúa los recursos disponibles para hacer frente a dicha situación. Si ambas apreciaciones no garantizan el éxito, es probable que se presenten cuadros de estrés.

El estrés es un fenómeno que forma parte de la rutina laboral en el mundo actual (Barron, 2004); produce bajo rendimiento, perdida de ciertas capacidades, agotamiento, inseguridad y accidentes laborales (Díaz, 2009); pone en riesgo la salud del colaborador y el correcto funcionamiento de la empresa (Moreno \& Báez, 2010). En el ámbito educativo, la dinámica laboral del docente genera un cúmulo de sensaciones, desgaste personal y emocional que puede producir estrés (Arís, 2009); las características actuales del estudiantado y la interacción con ellos son una de las principales fuentes de estrés del docente (Amador, Rodríguez, Serrano, Olvera \& Martínez, 2014); el ambiente de trabajo y la personalidad de los docentes condicionan la aparición de estrés y ansiedad (Martínez, 2003). Según Cardona (2010), las categorías que generan estrés en los docentes son, de mayor a menor impacto: la tecnología, el clima organizacional (percepción acerca de la organización), el territorio organizacional (espacio físico de trabajo), la estructura organizacional (relaciones jerárquicas entre colaboradores y jefes), falta de cohesión (problemas de unión en el equipo), influencia del líder (capacidad para influir) y el respaldo de grupo (apoyo en el logro de metas). Extremera, Rey y Pena (2010) consideran que los docentes presentan alto riesgo de padecer estrés y su sintomatología; todo indicaría que la enseñanza exige una mayor capacidad para hacer frente al estrés (Hiebert \& Farber, 1984).

Las exigencias psicológicas en el trabajo constituyen un factor de riesgo laboral que se presenta en diversos escenarios, y el trabajo docente no es la excepción. Los requerimientos asociados con la tranquilidad en el trabajo, el tiempo disponible y el empleo de habilidades físicas, cognitivas y emocionales, son un ejemplo de este tipo de exigencias. Rodríguez-Martínez, Tovalin-Ahumada, Gil-Monte, Salvador-Cruz y Acle-Tomasini (2018) encontraron que las condiciones estresantes y las exigencias emocionales se relacionan con la aparición de cuadros de ansiedad y depresión en docentes universitarios mexicanos. Ayuso (2006) considera que un horario poco adecuado dificulta el desarrollo de las actividades docentes; el exceso de horas de trabajo y un escenario exigente con fuertes demandas en competencias, conocimientos y 
habilidades, provocan que los docentes estén propensos al estrés (Barron, 2004). Pérez (2012) señala que las funciones administrativas encargadas a los docentes burocratizan su labor y los sobrecargan intelectualmente. Cuando las exigencias por cumplir las metas educativas superan el esfuerzo y las habilidades del docente, se presentan cuadros de insatisfacción, tensión y decrece la motivación laboral (Blase, 1982); experiencias emocionales negativas, relacionadas con la docencia, van acompañadas de estrés (Kyriacou, 2003).

El trabajo activo y las posibilidades de desarrollo representan un aspecto importante en la vida laboral del docente y se encuentran relacionados con la cantidad de trabajo, la posibilidad de aprender o desarrollar nuevas habilidades, el acceso a descansos durante la jornada y encontrar un sentido trascendente a la labor que realiza. Las presiones de tiempo y la carencia de materiales (Extremera et al., 2010), así como la asignación de horas en los tres turnos (mañana, tarde y noche) que obliga a los docentes a permanecer largos periodos de tiempo en la institución (González, 2008), son fuentes de estrés. Por la naturaleza del trabajo docente, Guerrero (2002) recomienda pequeños descansos para afrontar el estrés laboral. Cuando el docente percibe que no tiene libertad para desarrollar sus actividades y, si en un ambiente tóxico no tiene el control para modificar tal situación, se produce estrés, se presentan cuadros de ansiedad, irritabilidad y depresión (Barron, 2004).

Por otra parte, la claridad en los roles asignados, la posibilidad de aportar nuevos conocimientos o procedimientos que solucionan un problema, el apoyo de los jefes, las relaciones con los colegas y el liderazgo de los superiores en el manejo de conflictos son elementos que configuran un escenario de apoyo social al docente. La poca claridad en las funciones y sus respectivas responsabilidades, la carencia de criterios en la evaluación y las interacciones con colegas y estudiantes, pueden convertirse en factores que producen estrés (Guerrero, 2002); en el trabajo realizado por Caballero et al. (2009), un grupo de docentes manifestaron agotamiento físico y emocional como consecuencia de la interacción con estudiantes problemas. Las dificultades que se presentan entre colegas (Extremera et al., 2010), el poco apoyo entre pares (Barron, 2004) y el trabajo rutinario o repetitivo (González, 2008), pueden llevar a cuadros de estrés. Las presiones laborales ejercidas por los superiores sobre el trabajo docente (Oramas, Rodríguez, Almirall, Huerta \& Vergara, 2003) y una estructura rígida y vertical (Barron, 2004), también se convierten en situaciones que generan estrés en el docente.

Restrepo-Ayala, Colorado-Vargas y Cabrera-Arana (2006) señalan que la compensación es el factor más relevante en la aparición del estrés. Caballero et al. (2009) identificó que la falta de reconocimiento es un factor de riesgo psicosocial en los docentes universitarios que se acompaña de desgastes físico, indolencia e incremento del sentimiento de culpa. El poco reconocimiento, una imagen deteriorada (Martínez, 2003); organizaciones indiferentes donde no se toma en consideración la opinión del docente, trabajo inestable (Barron, 2004) y las expectativas laborales incumplidas (Extremera et al., 2010) se han convertido en impulsores de cuadros de estrés. Se presentan cuadros de tensión cuando los docentes no reciben los beneficios esperados, se frustran y la enseñanza que imparten se ve condicionada (González, 2008).

Según García, Iglesias, Saleta y Romay (2016), los docentes interinos y contratados son el grupo de trabajadores que perciben mayor inseguridad en el empleo. Luceño, Martín, Rubio y Jaén (2008) explican que los trabajadores con mayores factores de riesgo psicosociales y con mayores síntomas psicosomáticos (alteración del sueño, variación del apetito, cansancio, dolor de cabeza, dificultades para concertarse, entre otros) son aquellos que están expuestos a una menor estabilidad en el empleo o las recompensas que reciben son bajas o inestables. García et al. (2016) encontraron que la doble presencia (preocupación por cumplir las tareas del trabajo y al mismo tiempo, las tareas domésticas) afecta mayoritariamente a las mujeres; las responsabilidades domesticas se consideran fuentes de estrés para la enseñanza (Rojas \& Rodríguez, 2011). VallsLlobet (2000) explica como la doble jornada laboral, entre otros factores, ha convertido al trabajo doméstico en uno de los principales impulsores del estrés en las mujeres. 
El estudio de las percepciones de los docentes universitarios se convierte en piedra angular si se desea conocer las distintas variables que participan en el proceso de enseñanza-aprendizaje y en su desempeño profesional; por estas consideraciones, el objetivo central de la investigación es conocer la relación que existe entre los factores de riesgo psicosociales en el trabajo y el estrés percibido en los docentes universitarios, así mismo, se busca conocer las variaciones de esta relación según sexo, edad, tipo de universidad y modalidad de trabajo.

\section{Método}

\section{Diseño de la investigación}

Por el tratamiento estadístico de los datos, la investigación presenta un enfoque cuantitativo, de alcance correlacional, con un diseño no experimental y transversal. Es un estudio correlacional porque se asocian dos variables (factores de riesgo psicosociales y estrés percibido) y se busca conocer la relación entre ellas; el diseño es no experimental debido a que no se han manipulado las variables, y es transversal porque los datos fueron recogidos en un solo momento (Hernández, Fernández \& Baptista, 2014).

\section{Participantes}

La muestra estuvo conformada por 117 docentes universitarios de la ciudad Lima, del departamento de Lima, Perú. Las universidades, en las cuales laboran los docentes participantes, pertenecen a un NSE medio. Se tuvo como criterios de inclusión: (1) trabajar a tiempo completo en la universidad y (2) participar voluntariamente en la investigación. La muestra presentó una edad promedio de 45 años $(D E=5.86)$, con edad mínima y máxima 35 y 54 años, respectivamente. El 52\% de la muestra eran hombres y el $48 \%$ mujeres, $54.7 \%$ pertenecían a la universidad privada, mientras que el $45.3 \%$ a la universidad pública. Además, el $67.5 \%$ de los docentes laboraban bajo la condición de contratados, mientras que el $32.5 \%$ bajo la condición de nombrados. De los participantes, todos los docentes que pertenecían a la universidad privada poseían la condición de contratados, por el contrario, el $28.3 \%$ de los docentes que pertenecían a la universidad pública eran contratados, mientras que el $71.7 \%$ eran nombrados.

\section{Instrumentos}

SUSESO-ISTAS 21 (Superintendencia de Seguridad Social, 2018). Para medir la variable factores de riesgo psicosociales en el trabajo se empleó el test SUSESO-ISTAS 21 versión breve, el cual fue elaborado por requerimiento de la Superintendencia de Seguridad Social de Chile; este instrumento es una adaptación al español del Copenhagen Psychosocial Questionnaire (COPSOQ) elaborado por el Instituto Nacional de Seguridad y Salud Laboral de Dinamarca (Superintendencia de Seguridad Social, 2018). El SUSESO-ISTAS 21 versión breve presenta cinco dimensiones: Exigencias psicológicas, Trabajo activo y Desarrollo de habilidades, Apoyo social y liderazgo en la empresa, Compensaciones y Doble presencia; las cuales se miden a través de 20 ítems, con escala tipo Likert de cuatro opciones de respuesta, donde cero significa «Nunca» y cuatro «Siempre».

Con el objetivo de demostrar validez de constructo, se realizó un análisis factorial exploratorio al instrumento. Se confirmó que los 20 ítems de la escala se agrupan en una estructura de cinco dimensiones, las cuales explican el $60 \%$ de la variabilidad de las respuestas. Además, se puede observar en la tabla 1 que las cargas factoriales de cada ítem fueron superiores a .50.

En la presente investigación, se aplicó la prueba de confiabilidad a través del alfa de Cronbach a la escala general y a las cinco dimensiones del SUSESO-ISTAS 21, en la tabla 2 se observa que los valores obtenidos son iguales o superiores a .67, lo que indicaría que el instrumento es confiable. También se puede apreciar los intervalos de confianza al 95\% para cada valor de alfa de Cronbach. 
Tabla 1.

Solución factorial del SUSESO-ISTAS 21 versión breve

\begin{tabular}{|c|c|c|c|c|c|}
\hline $\begin{array}{l}\mathrm{N}^{\circ} \text { de } \\
\text { Ítem }\end{array}$ & $\begin{array}{l}\text { Exigencias } \\
\text { Psicológicas }\end{array}$ & $\begin{array}{c}\text { Trabajo } \\
\text { Activo y } \\
\text { Desarrollo } \\
\text { de } \\
\text { Habilidades }\end{array}$ & $\begin{array}{c}\text { Apoyo } \\
\text { Social y } \\
\text { Liderazgo } \\
\text { en la } \\
\text { Empresa }\end{array}$ & Compensaciones & $\begin{array}{c}\text { Doble } \\
\text { Presencia }\end{array}$ \\
\hline 1 & .65 & & & & \\
\hline 2 & .65 & & & & \\
\hline 3 & .64 & & & & \\
\hline 4 & .57 & & & & \\
\hline 5 & .75 & & & & \\
\hline 6 & & .75 & & & \\
\hline 7 & & .71 & & & \\
\hline 8 & & .77 & & & \\
\hline 9 & & .62 & & & \\
\hline 10 & & .83 & & & \\
\hline 11 & & & .66 & & \\
\hline 12 & & & .76 & & \\
\hline 13 & & & .63 & & \\
\hline 14 & & & .70 & & \\
\hline 15 & & & .76 & & \\
\hline 16 & & & & .64 & \\
\hline 17 & & & & .58 & \\
\hline 18 & & & & .51 & \\
\hline 19 & & & & & .80 \\
\hline 20 & & & & & .86 \\
\hline
\end{tabular}

Tabla 2.

Índices de confiabilidad del SUSESO-ISTAS 21 versión breve

\begin{tabular}{cccc}
\hline & \multirow{2}{*}{$\begin{array}{c}\text { Alfa de } \\
\text { Cronbach }\end{array}$} & \multicolumn{2}{c}{ Intervalo de confianza al 95\% } \\
\cline { 3 - 4 } & & $\begin{array}{c}\text { Límite } \\
\text { inferior }\end{array}$ & $\begin{array}{c}\text { Límite } \\
\text { superior }\end{array}$ \\
\hline Escala General & .70 & .61 & .78 \\
Exigencias Psicológicas & .68 & .58 & .77 \\
Trabajo Activo y Desarrollo de Habilidades & .83 & .78 & .88 \\
Apoyo Social y Liderazgo en la Empresa & .76 & .68 & .82 \\
Compensaciones & .67 & .57 & .76 \\
Doble Presencia & .72 & .63 & .80 \\
\hline
\end{tabular}

Escala Estrés Percibido: PSS14 (González \& Landero, 2007). Para medir la segunda variable, se utilizó la adaptación mexicana denominada Escala de Estrés Percibido PSS14 (González \& Landero, 2007). Este instrumento está basado en el test elaborado por Cohen y colaboradores en 1983, que a su vez parte de la definición de estrés psicológico propuesto por Lazarus y Folkman (Cohen, Kamarck \& Mermelstein, 1983). Este test busca medir el grado en el cual los individuos valoran como estresantes las situaciones del último mes. El PSS14 consta de 14 ítems con formato de respuesta tipo Likert de cinco opciones, las cuales van desde cero (nunca) a cuatro (siempre). Asimismo, el instrumento cuenta con siete ítems $(4,5,6,7,9,10$ y 13$)$ que se deben invertir para calcular el puntaje total. 
Se realizó un análisis factorial exploratorio al instrumento con el propósito de demostrar la validez de constructo. En el análisis se confirmó la estructura unidimensional del instrumento, la cual explica el $30 \%$ de la variabilidad de las respuestas. Asimismo, las cargas factoriales de cada ítem fueron iguales o superiores a .47 (ver tabla 3 ).

Tabla 3.

Solución Factorial de la escala PSS14

\begin{tabular}{cc}
\hline $\mathrm{N}^{\circ}$ de Ítem & Carga Factorial \\
\hline 1 & .61 \\
2 & .47 \\
3 & .58 \\
4 & .48 \\
5 & .57 \\
6 & .59 \\
7 & .50 \\
8 & .60 \\
9 & .52 \\
10 & .56 \\
11 & .58 \\
12 & .57 \\
13 & .55 \\
14 & .47 \\
\hline
\end{tabular}

En la adaptación mexicana del PSS14 de González y Landero (2007) se obtuvo como consistencia interna un índice de alfa de Cronbach de .83; en la presente investigación, el alfa de Cronbach presentó un índice de .82, con intervalos de confianza al 95\% de .76 (límite inferior) a .87 (límite suprior); lo anterior demuestra que el instrumento es confiable.

Síntomas de estrés. Hiebert y Farber (1984) analizaron diversos estudios sobre el estrés y explicaron que la profesión docente podría llegar a ser estresante y que la percepción del estrés se ve condicionada a las exigencias del medio y a los recursos para afrontarlo. Resaltan que los resultados no son concluyentes para establecer que la enseñanza sea una actividad estresante, pero tampoco alcanzan para afirmar que no lo sea, por lo que recomiendan realizar más investigaciones sobre el tema. Extremera et al. (2010) analizaron los síntomas asociados al estrés en docentes de primaria y secundaria de la provincia de Huelva, España; encontraron que ambos grupos comparten síntomas como: cansancio, dificultades con el sueño, dolor de cabeza, entre otros. En una de sus recomendaciones, los autores sugieren recoger datos en otro grupo docente, por ejemplo, los que se dedican a la docencia universitaria, para obtener mayor evidencia y generalizar los resultados.

De acuerdo con lo anterior, se quiso profundizar en el estudio sintomatológico del estrés en docentes universitarios, por ello se adicionó la pregunta ¿Cuáles son los principales síntomas que experimenta cuando está estresado? La ficha que contenía esta interrogante llevaba como introducción la definición de estrés propuesta Lazarus y Folkman (1986), lo que permitía al docente evaluado reportar por escrito las manifestaciones fisiológicas producidas por el estrés que creía experimentar.

\section{Procedimiento}

Para la selección de la muestra se empleó un muestreo no probabilístico: muestreo por conveniencia; el cual permite al investigador tomar en cuenta los casos disponibles a los que se tienen acceso (Hernández et al., 2014). De esta forma, la muestra estuvo conformada por 117 docentes universitarios que aceptaron participar de forma voluntaria en la investigación y que laboraban a tiempo completo. Los investigadores entregaron a los participantes un cuadernillo, el 
cual contenía el consentimiento informado, la ficha sociodemográfica, el SUSESO-ISTAS 21, el PSS14 y la ficha con la pregunta sobre la sintomatología. En el consentimiento informado se indicó los objetivos de la investigación, la duración de las pruebas, su derecho a rehusarse a participar, finalizar el cuestionario o retirarse del estudio; también se informó sobre la participación anónima y que sus datos serían usados únicamente para fines del estudio. Por otro lado, la ficha sociodemográfica permitió obtener información tal como: sexo, edad, tipo de universidad (privada y pública) y modalidad de trabajo (contratado y nombrado). El llenado de los documentos duró en promedio 25 minutos por persona y se realizó en las aulas de cada universidad durante los tiempos libres de los docentes. La recolección de datos se llevó a cabo a finales del 2018 en diferentes universidades de Lima, Perú.

\section{Análisis de datos}

Para analizar los datos se utilizó el paquete estadístico SPSS Statistics versión 22.0. Primero se realizó un análisis de identificación de casos atípicos, se encontraron dos casos con puntuaciones que se alejaban extremadamente de la media. Sin embargo, se decidió no eliminarlos, ya que los casos formaban parte de la diversidad de respuesta de la muestra, además de no generar variación significativa en los resultados.

Se empleó la prueba Shapiro-Wilk para evaluar la normalidad de las puntuaciones obtenidas en cada prueba (ver tabla 4). Se encontró falta de normalidad en todas las puntuaciones, excepto en los datos del estrés percibido.

Tabla 4

Prueba de normalidad: Shapiro-Wilk

\begin{tabular}{ccccc}
\hline & $M$ & $D E$ & W & Sig. \\
\hline Exigencias & 17.96 & 1.78 & .90 & .001 \\
psicológicas & & & & \\
Trabajo activo & 16.79 & 2.81 & .89 & .001 \\
Apoyo social & 3.75 & 2.51 & .95 & .001 \\
Compensaciones & 10.32 & 1.46 & .87 & .001 \\
Doble presencia & 7.20 & 1.02 & .77 & .001 \\
Estrés percibido & 46.46 & 4.86 & .98 & .076 \\
\hline
\end{tabular}

En el análisis de los coeficientes de asimetría $(<|3|)$ y curtosis $(<|8|)$, se concluyó que no existía falta de normalidad severa (Kline, 2005), con estos resultados se emplearon los análisis paramétricos correspondientes. Se realizó el análisis correlacional con el coeficiente $r$ de Pearson entre las variables de estudio. Adicionalmente, las variables fueron correlacionadas según las características sociodemográficas, con el propósito de conocer bajo qué condiciones (sexo: mujeres u hombres, edad: 35-44 o 45-54, tipo de universidad: privada o pública, y modalidad de trabajo: contratados o nombrados) las correlaciones podrían presentar variaciones. Por último, se realizaron contrastes de medias utilizando la prueba paramétrica $t$ de Student según las características sociodemográficas.

\section{Resultados}

Para responder el objetivo central de la investigación, se correlacionaron las variables de estudio; se observó correlaciones positivas y medianas entre el estrés percibido y las exigencias psicológicas, compensaciones y doble presencia. Además, se encontró que la correlación entre el estrés percibido y trabajo activo fue positiva y pequeña. Por otro lado, se evidenció una relación inversa de tamaño mediano entre la dimensión apoyo social y la variable estrés percibido. Además, en la tabla 5 se pueden apreciar los intervalos de confianza. 
Tabla 5.

Correlaciones entre las variables de estudio

\begin{tabular}{cccc}
\hline & \multirow{2}{*}{ Estrés percibido } & \multicolumn{2}{c}{ Intervalo de confianza al 95\% } \\
\cline { 3 - 4 } & $.37^{* * *}$ & Límite inferior & Límite superior \\
\hline Exigencias psicológicas & $.20^{*}$ & .20 & .51 \\
Trabajo activo & $-.32^{* * *}$ & .02 & .37 \\
Apoyo social & $.38^{* * *}$ & -.47 & -.15 \\
Compensaciones & $.42^{* * *}$ & .22 & .53 \\
Doble presencia & .26 & .56 \\
\hline
\end{tabular}

$* p<.05 ; * * p<.01 ; * * * p<.001$

Al correlacionar las variables de estudio según el tipo de universidad donde laboran los docentes, se halló que todas las relaciones correspondientes a la universidad privada reportaron un mayor índice de correlación en comparación con los resultados de los docentes de la universidad pública. En los docentes de universidad privada, las correlaciones entre el estrés percibido con las exigencias psicológicas y las compensaciones fueron positivas y grandes. Según Cohen (1988), los valores q menores a .10 representan una diferencia de tamaño insignificante entre las correlaciones, entre .10 y .30 de tamaño pequeño, entre .31 y .50 de tamaño mediano y mayores a .51 de tamaño grande. $\mathrm{Al}$ analizar la q de Cohen se aprecia que las diferencias entre las correlaciones del estrés percibido con las exigencias psicológicas, el apoyo social y las compensaciones presentaron un tamaño pequeño.

Tabla 6.

Correlaciones entre las variables de estudio según el tipo de universidad

\begin{tabular}{cccc}
\hline & \multicolumn{2}{c}{ Estrés percibido } & \multirow{2}{*}{$q$} \\
\cline { 2 - 3 } & U. Privada & U. Pública & .12 \\
Exigencias psicológicas & $.53^{* * *}$ & $.44^{* *}$ & .05 \\
Trabajo activo & $.42^{* *}$ & $.38^{* *}$ & .10 \\
Apoyo social & $-.37^{* *}$ & $-.28^{*}$ & .20 \\
Compensaciones & $.53^{* * *}$ & $.37 * *$ & .02 \\
Doble presencia & $.48^{* * *}$ & $.45^{* *}$ &
\end{tabular}

$* p<.05 ; * * p<.01 ; * * * \mathrm{p}<.001$

Según la modalidad de trabajo, en comparación con los docentes nombrados, los docentes contratados presentan correlaciones mayores entre las variables de estudio (ver tabla 7). Siendo estas diferencias de tamaño pequeño (q de Cohen con valores entre .10 y .30). No se encontraron correlaciones significativas en cuanto a la edad y al sexo.

Tabla 7.

Correlaciones entre las variables de estudio según modalidad de trabajo

\begin{tabular}{cccc}
\hline & \multicolumn{2}{c}{ Estrés percibido } & \multirow{2}{*}{$q$} \\
\cline { 2 - 3 } & Contratados & Nombrados & \\
\hline Exigencias psicológicas & $.49^{* *}$ & $.38^{*}$ & .14 \\
Trabajo activo & $.42^{* *}$ & $.34^{*}$ & .10 \\
Apoyo social & $-.38^{* *}$ & -.13 & .27 \\
Compensaciones & $.50^{* *}$ & $.35^{*}$ & .17 \\
Doble presencia & $.48^{* *}$ & $.39^{*}$ & .11 \\
\hline
\end{tabular}

${ }^{*} p<.05 ; * * \mathrm{p}<.001$

Por otro lado, se realizaron comparaciones de medias según las características sociodemográficas de los docentes. En cuanto al tipo de universidad, en la tabla 8 se aprecia que los docentes que provienen de la universidad con gestión privada presentan mayores medias en las dimensiones exigencias psicológicas, trabajo activo, compensaciones y doble presencia. 
Según Cohen (1988) una d con un valor inferior a 0.20 significa una diferencia insignificante, de 0.21 a 0.50 una diferencia pequeña, de 0.51 a 0.80 una diferencia mediana, y superior a 0.80 señala una diferencia grande. Las diferencias entre las medias de las dimensiones exigencias psicológicas y trabajo activo presentan una d de Cohen superior a 0.80 , esto indicaría que las diferencias son grandes y bastante perceptibles; en las compensaciones y la doble presencia, las diferencias entre las medias son de tamaño mediano. Por el contrario, las medias en la dimensión apoyo social presentan una diferencia insignificante (ver tabla 8).

Tabla 8.

Prueba t de la variable factores de riesgo psicosociales según tipo de universidad

\begin{tabular}{|c|c|c|c|c|c|c|}
\hline & \multicolumn{2}{|c|}{$\begin{array}{l}\text { U. Privada } \\
\quad(n=64)\end{array}$} & \multicolumn{2}{|c|}{$\begin{array}{l}\text { U. Pública } \\
(n=53)\end{array}$} & \multirow[t]{2}{*}{$t$} & \multirow[t]{2}{*}{$d$} \\
\hline & $M$ & $D E$ & $M$ & $D E$ & & \\
\hline Exigencias psicológicas & 18.80 & 1.31 & 16.94 & 1.75 & $6.55 * *$ & 1.20 \\
\hline Trabajo activo & 18.69 & 1.54 & 14.51 & 2.25 & $11.47 * *$ & 2.17 \\
\hline Apoyo social & 3.63 & 2.79 & 3.91 & 2.14 & -0.60 & 0.11 \\
\hline Compensaciones & 10.81 & 1.37 & 9.74 & 1.35 & $4.27 * *$ & 0.79 \\
\hline Doble presencia & 7.44 & 0.94 & 6.91 & 1.04 & $2.90 *$ & 0.53 \\
\hline
\end{tabular}

$* p<.01 ; * * \mathrm{p}<.001$

En relación con la modalidad de trabajo, se observó mayores medias en las exigencias psicológicas, trabajo activo y compensaciones de los docentes contratados. Las diferencias entre las medias (d de Cohen) de las exigencias psicológicas y compensaciones son de tamaño mediano; mientras que la diferencia de medias del trabajo activo es grande, lo cual indicaría que es una diferencia bastante perceptible (ver tabla 9). Por otro lado, no se reportó diferencias según edad y sexo.

Tabla 9.

Prueba t de la variable factores de riesgo psicosociales según modalidad de trabajo

\begin{tabular}{|c|c|c|c|c|c|c|}
\hline & \multicolumn{2}{|c|}{$\begin{array}{c}\text { Contratados } \\
(n=79)\end{array}$} & \multicolumn{2}{|c|}{$\begin{array}{c}\text { Nombrados } \\
(n=38)\end{array}$} & \multirow[t]{2}{*}{$t$} & \multirow[t]{2}{*}{$d$} \\
\hline & $M$ & $D E$ & $M$ & $D E$ & & \\
\hline $\begin{array}{l}\text { Exigencias } \\
\text { psicológicas }\end{array}$ & 18.33 & 1.72 & 17.18 & 1.67 & $3.41 * *$ & 0.68 \\
\hline Trabajo activo & 17.90 & 2.34 & 14.5 & 2.3 & $7.40 * * *$ & 1.47 \\
\hline Apoyo social & 3.85 & 2.67 & 3.55 & 2.15 & 0.60 & 0.12 \\
\hline Compensaciones & 10.56 & 1.43 & 9.84 & 1.41 & $2.55^{*}$ & 0.51 \\
\hline Doble presencia & 7.25 & 0.99 & 7.08 & 1.08 & 0.86 & 0.16 \\
\hline
\end{tabular}

$*_{p}<.05 ; * * p<.01 ; * * * \mathrm{p}<.001$

En el caso de la variable estrés percibido, según la prueba t solo se encontraron diferencias según la modalidad de trabajo, siendo los docentes contratados el grupo que obtiene mayor puntaje en el estrés percibido. Asimismo, en la tabla 10 se aprecia que el tamaño de la diferencia entre las medias es pequeño (0.45).

Tabla 10.

Prueba t de la variable estrés percibido según modalidad de trabajo

\begin{tabular}{ccccc}
\hline & $M$ & $D E$ & $t$ & $d$ \\
\hline Nombrados $(n=38)$ & 45.77 & 4.809 & $-2.25^{*}$ & \multirow{2}{*}{0.45} \\
Contratados $(n=79)$ & 47.89 & 4.701 & & \\
\hline
\end{tabular}

$* p<.05$ 
Adicionalmente, se reportó que los principales síntomas que experimentan los docentes son: estar cansado, no poder dormir y presentar dolores de cabeza (ver tabla 11).

Tabla 11.

Principales síntomas de estrés experimentados

$\%$

Me siento cansado todo el tiempo

45.2

No puedo dormir

36.1

Me duele la cabeza

32.4

No puedo concentrarme

28.0

Pierdo el apetito o como más de lo normal

26.3

Estoy irritado todo el tiempo

23.1

Me enfermo todo el tiempo

18.1

Estoy ansioso todo el tiempo

16.2

Bebo o fumo más de lo normal

9.2

Otros

10.0

Un mayor porcentaje de mujeres, en comparación con los hombres, reportan que han experimentado problemas para dormir, dolores de cabeza, falta de concentración, variaciones en el apetito, irritabilidad y ansiedad. En cuanto a la edad, se ha reportado que los docentes entre 45 y 54 años experimentan con mayor frecuencia cansancio, problemas para dormir, dolores de cabeza, irritabilidad, sentirse enfermo y ansiedad (ver tabla 12).

Tabla 12.

Principales síntomas de estrés según sexo y edad

\begin{tabular}{cccccc}
\hline & Total & \multicolumn{2}{c}{ Sexo } & \multicolumn{2}{c}{ Edad } \\
\cline { 3 - 6 } & $(\%)$ & Mujeres & Hombres & $35-44$ & $45-54$ \\
& & $(\%)$ & $(\%)$ & $(\%)$ & $(\%)$ \\
\hline Me siento cansado todo el tiempo & 45.2 & 41.6 & 48.8 & 39.4 & 51.0 \\
No puedo dormir & 36.1 & 39.8 & 32.5 & 33.0 & 39.2 \\
Me duele la cabeza & 32.4 & 34.7 & 30.1 & 30.8 & 34.0 \\
No puedo concentrarme & 28.0 & 35.1 & 20.9 & 31.0 & 25.0 \\
Pierdo el apetito o como más de lo & 26.3 & 32.2 & 20.4 & 32.1 & 20.5 \\
normal & 23.1 & 25.2 & 21.0 & 18.0 & 28.3 \\
Estoy irritado todo el tiempo & 18.1 & 12.1 & 23.9 & 15.1 & 21.0 \\
Me enfermo todo el tiempo & 16.2 & 18.0 & 14.4 & 13.2 & 19.2 \\
Estoy ansioso todo el tiempo & 9.2 & 5.9 & 12.5 & 10.3 & 8.1 \\
Bebo o fumo más de lo normal & 10.0 & 9.0 & 11.0 & 8.0 & 12.0 \\
Otros & & & & &
\end{tabular}

Se puede apreciar que existe un mayor porcentaje de percepción de síntomas de estrés por parte de los docentes que laboran la universidad privada, así como en los docentes que son contratados (ver tabla 13).

Tabla 13.

Principales síntomas de estrés según tipo de universidad y modalidad de trabajo

\begin{tabular}{cccccc}
\hline & \multirow{2}{*}{$\begin{array}{c}\text { Total } \\
(\%)\end{array}$} & \multicolumn{2}{c}{$\begin{array}{c}\text { Tipo de } \\
\text { universidad }\end{array}$} & \multicolumn{2}{c}{ Modalidad de trabajo } \\
\cline { 3 - 6 } & & $\begin{array}{c}\text { Privada } \\
(\%)\end{array}$ & $\begin{array}{c}\text { Pública } \\
(\%)\end{array}$ & $\begin{array}{c}\text { Contratados } \\
(\%)\end{array}$ & $\begin{array}{c}\text { Nombrados } \\
(\%)\end{array}$ \\
\hline $\begin{array}{c}\text { Me siento cansado todo el } \\
\text { tiempo }\end{array}$ & 45.2 & 51.1 & 39.3 & 51.2 & 39.2
\end{tabular}




\begin{tabular}{cccccc} 
No puedo dormir & 36.1 & 39.2 & 33.0 & 39.0 & 33.2 \\
Me duele la cabeza & 32.4 & 36.5 & 28.3 & 37.1 & 27.7 \\
No puedo concentrarme & 28.0 & 31.0 & 25.0 & 33.0 & 23.0 \\
Pierdo el apetito o como más & 26.3 & 32.5 & 20.1 & 33.4 & 19.2 \\
de lo normal & & 27.8 & 18.4 & 27.3 & 18.9 \\
Estoy irritado todo el tiempo & 23.1 & 21.2 & 15.0 & 20.4 & 16.0 \\
Me enfermo todo el tiempo & 18.1 & 21.3 & 11.1 & 19.2 & 13.2 \\
Estoy ansioso todo el tiempo & 16.2 & 11.1 & 7.3 & 8.4 & 10.0 \\
Bebo o fumo más de lo & 9.2 & 11.0 & 13.0 & 7.0 \\
normal & 10.0 & 12.0 & 8.0 & & \\
Otros & & & & & \\
\hline
\end{tabular}

\section{Discusión}

Según los resultados, las exigencias psicológicas, las compensaciones y la doble presencia son factores de riesgo psicosociales asociados al trabajo que podrían generar estrés en el docente universitario; estos hallazgos concuerdan con los planteamientos de Gil-Monte (2012), Raffo et al. (2013), Carlotto y Gonçalves (2017), Barron (2004), Arís (2009), Amador et al. (2014), Martínez (2003), Cardona (2010), Ayuso (2006), Pérez (2012), Kyriacou (2003), Extremera et al. (2010), Guerrero (2002), González (2008), Oramas et al. (2003), Restrepo-Ayala et al. (2006), Caballero et al. (2009), Rojas y Rodríguez (2011) y Rodríguez-Martínez et al. (2018). Por otro lado, la dimensión trabajo activo obtuvo una correlación de .20 con el estrés percibido y el límite inferior del intervalo de confianza mostró un índice de .02 , esto indica la probabilidad de una correlación nula. Por lo anterior, es necesario tomar con precaución el índice de correlación entre el trabajo activo y el estrés percibido.

Si bien los índices de correlación en los docentes de la universidad pública son importantes, los resultados indican que las correlaciones entre los factores de riesgo psicosociales y el estrés son mayores en los docentes de la universidad privada, especialmente las dimensiones exigencias psicológicas (exigencias emocionales, creativas, sensoriales y cantidad y ritmo de trabajo) y compensaciones (estabilidad en el empleo y cambios no deseados). Estos resultados reflejarían que las exigencias físicas, académicas y emocionales de las instituciones de gestión privada se han convertido en factores de riesgo psicosociales que alimentan el estrés percibido por el docente, por otra parte, el tema de las compensaciones es una arista sensible y los resultados expuestos se podrían relacionar con la ratificación del personal contratado, la renovación semestral o anual de los docentes, los cambios de horario y carga académica que depende del número de matrículas. Sería importante buscar diversos incentivos, por ejemplo, un salario emocional, para que los docentes no perciban estas exigencias, válidas dentro de un mundo competitivo que busca la excelencia académica y brindar un servicio de calidad, como factores de riesgo psicosociales que los llevarían al estrés.

Por otra parte, la correlación general entre el apoyo social y el estrés percibido es inversa, además, según el análisis de resultados entre los tipos de universidades, también se observó correlaciones en sentido inverso en docentes de la universidad privada y docentes de la universidad pública. Esto indicaría que el apoyo recibido por los colegas y jefes, la calidad en el liderazgo de los superiores, la asignación clara de funciones, la calidad en las relaciones con los colegas y el acceso a información oportuna para el desarrollo de las actividades, no representan un factor de riesgo psicosocial, es decir, un adecuado apoyo social contribuiría a disminuir la percepción de estrés del docente e inclusive podría considerarse como un moderador de los efectos nocivos de aquellos factores que son percibidos como riesgos psicosociales. De acuerdo con lo anterior, se debería reforzar el apoyo social a los docentes universitarios; la gestión académica y administrativa de las universidades deberían considerar estos aspectos para ofrecer un entorno emocional y socialmente agradable que permita desarrollar saludablemente todas las actividades ligadas a la profesión docente y, de esta forma, evitar posibles cuadros de estrés. 
En cuanto a la modalidad de trabajo, las correlaciones entre los factores de riesgo psicosociales (exigencias psicológicas, trabajo activo, compensaciones y doble presencia) y el estrés percibido son mayores en los docentes contratados. La correlación es inversa entre el apoyo social y el estrés percibido; además, los docentes contratados son los que perciben mayor nivel de estrés, estos hallazgos parecen concordar con lo expuesto por García et al. (2016) y Luceño et al. (2008). Es probable que los resultados de esta investigación sean el reflejo de la problemática que se vive en el país a razón de los nuevos cambios y exigencias académicas, legales y administrativas; es posible que los docentes contratados perciban mayores factores de riesgo psicosociales en el trabajo debido a que su continuidad depende de muchos factores, entre ellos: el desempeño docente, evaluación de satisfacción de los estudiantes, elaboración y presentación de documentos, evaluación de los coordinadores, exigencia de capacitación continua, participar activamente en el desarrollo de investigaciones, asistencias a conferencias, congresos, seminarios, etc. Esto sin olvidar que, posiblemente, los docentes contratados no se dedican exclusivamente a las labores académicas, también habría que considerar otras responsabilidades.

También es relevante comentar que los docentes nombrados, que provienen de la universidad pública, gozan de ciertos beneficios a diferencia de los contratados, por ejemplo: remuneración mensual integra, bonificación extra en caso de lograr algún cargo administrativo, beneficios sociales completos, seguridad de continuidad en la institución (previa evaluación), mayor cantidad de días de descanso, etc.; estas condiciones podrían explicar las diferencias en la percepción de los factores de riesgo psicosociales y el estrés en los docentes contratados y nombrados. Como se mencionó anteriormente, es válido que las universidades, sin distinción alguna, exijan calidad académica y profesional a los docentes, pero deberían girar la mirada a cómo percibe el docente el entorno donde trabaja y ver el impacto que tiene en su desempeño y salud.

En cuanto a la dimensión de doble presencia, la presente investigación no ha encontrado diferencia significativa entre varones y mujeres, esto no coincide con los resultados de VallsLlobet (2000) y García et al. (2016). Según la literatura consultada, la preocupación por las exigencias domesticas afecta, principalmente, el desempeño laboral de las mujeres; sin embargo, es posible que los participantes de este estudio compartan dichas responsabilidades, lo cual lleva a pensar que las diferencias de género, en cuanto a actividades domésticas y familiares se refiere, van disminuyendo.

Los cinco síntomas de estrés más comunes reportados por los participantes fueron: cansancio, no poder dormir, dolores de cabeza, falta de concentración y cambios en el apetito; este reporte parece coincidir los aportes de Moreno y Báez (2010), Pérez (2012), Díaz (2009) y Extremera et al. (2010). Las exigencias académicas, físicas y emocionales pueden provocar falta de fuerzas para seguir la jornada. Dormir es una actividad necesaria para recuperar energías y permitir que el cuerpo repare tejidos, su carencia puede llevar a padecer distintas enfermedades. La tensión acumulada puede causar dolor de cabeza y problemas cognitivos relacionados a la atención y una deficiente alimentación puede ocasionar daños severos a la salud. Estos síntomas indicarían que los docentes encuestados han manifestados cuadros de estrés, afectando su salud y desempeño profesional; parece que lo anterior coincide con lo propuesto por Hiebert y Farber (1984), la profesión docente puede llegar a ser estresante.

De todos los participantes, las mujeres, los docentes de 45 a 54 años, los docentes de universidades privadas y aquellos que tienen la condición de contratados, reportan mayor sintomatología; lo anterior indicaría que estos grupos presentan mayor vulnerabilidad ante las demandas del entorno. En el caso de las mujeres, según Duval, González y Rabia (2010), esta condición encontraría respuesta en las diferencias neurobiológicas y en el papel que juega la hormona corticotropina (CRF). En el caso de los docentes mayores, estos se podrían ver afectados por las "nuevas" exigencias emocionales y tecnológicas que requiere el proceso de enseñanza en las universidades, a esto también se suma las características particulares de los estudiantes nacidos en la era digital. 
El estudio, al tener un alcance correlacional, no ha podido determinar la causalidad entre los factores de riesgo psicosociales y la percepción del estrés en los docentes universitarios, sin embargo, los resultados de la presente investigación sirven para profundizar en el conocimiento de las percepciones docentes y su posible impacto en la salud y desempeño profesional. Si bien la pregunta sobre la sintomatología resultó importante para analizar la realidad del docente universitario, es importante reconocer que dicho diagnóstico no se sustenta en pruebas objetivas ni análisis médicos especializados, por lo que sería importante implementarlos en futuras investigaciones.

Así mismo, por la naturaleza del diseño de investigación y de los instrumentos empleados, sería recomendable promover más investigaciones en este campo de estudio, pero desde el punto de vista cualitativo. El empleo de técnicas cualitativas, como las entrevistas personalizadas y a profundidad, así como el focus group, son procedimientos validos que servirían para profundizar en aquellos aspectos que no se han podido dilucidar con la información cuantitativa, pero que servirían para valorar el trabajo docente y ofrecer un ambiente agradable y seguro donde enseñar.

\section{Referencias}

Amador, R., Rodríguez, C., Serrano, J., Olvera, J., \& Martínez, S. (2014). Estrés y burnout en docentes de educación media superior. Revista Electrónica Medicina, Salud y Sociedad, 4(2), 119-141. Recuperado de http://ri.uaemex.mx/handle/20.500.11799/32405

Arís, N. (2009) El Síndrome de Burnout en los docentes. Revista electrónica de investigación psicoeducativa, 7(18), 829-848. doi. http://dx.doi.org/10.25115/ejrep.v7i18.1324

Ayuso, J. (2006). Profesión docente y estrés laboral: una aproximación a los conceptos de Estrés Laboral y Burnout. Revista Iberoamericana De Educación, 39(3), 1-15. doi: https://doi.org/10.35362/rie3932575

Barron, J. (2004). Factores o causas que generan posibles fuentes de estrés en el ámbito laboral docente en una institución educativa. Población: Universidad Nacional de Río Cuarto. Kairos, 3(13). Recuperado de http://www.revistakairos.org/wpcontent/uploads/barron.pdf

Blase, J. (1982). A Social-psychological grounded theory of teacher stress and burnout. Educational Administration Quarterly, 18(4), 93-113. doi: https://doi.org/10.1177/0013161X82018004008

Caballero, C., González, O., Mercado, D., Llanos, C., Bermejo, Y., \& Vergel, C. (2009). Prevalencia del síndrome del burnout y su correlación con factores psicosociales en docentes de una institución universitaria privada de la ciudad de Barranquilla. Psicogente, 12(21), 142-157. Recuperado de http://revistas.unisimon.edu.co/index.php/psicogente/article/view/1192

Caballero-Lozada, M., \& Nieto, L. (2015). Nueva gestión pública en Colombia y bienestar laboral del profesorado universitario. Entramado, 11(1), 124-134. doi: http://dx.doi.org/10.18041/entramado.2015v11n1.21105

Cardona, L. (2010). Estrés laboral en docentes de la facultad de ingenierías de la Universidad Libre de Pereira. Cultura del Cuidado Enfermería, 7(2), 28-38. Recuperado de https://revistas.unilibre.edu.co/index.php/cultura/article/view/3779

Carlotto, M., \& Gonçalves, S. (2017). Riesgos psicosociales asociados con el síndrome de burnout en profesores universitarios. Avances en Psicología Latinoamericana, 35(3), 447-457. doi: http://dx.doi.org/10.12804/revistas.urosario.edu.co/apl/a.4036

Cohen J. (1988). Statistical Power Analysis for the Behavioral Sciences. New York, EEUU: Lawrence Erlbaum Associates.

Cohen, S., Kamarck, T., \& Mermelstein, R. (1983). A global measure of perceived stress. Journal of Health and Social Behavior, 24(4), 385-396. doi: http://dx.doi.org/10.2307/2136404

Díaz, P. (2009). Prevención de riesgos laborales: seguridad y salud laboral. Madrid: Editorial Paraninfo.

Duval, F., González, F., \& Rabia, H. (2010). Neurobiología del estrés. Revista chilena de neuropsiquiatría, 48(4), 307-318. doi: https://dx.doi.org/10.4067/S0717-92272010000500006 
Extremera, N., Rey, L., \& Pena, M. (2010). La docencia perjudica seriamente la salud. Análisis de los síntomas asociados al estrés docente. Boletín de psicología, (100), 43-54. Recuperado de https://www.uv.es/seoane/boletin/previos/N100-3.pdf

García, M., Iglesias, S., Saleta, M., \& Romay, J. (2016). Riesgos psicosociales en el profesorado de enseñanza universitaria: diagnóstico y prevención. Revista de psicología del trabajo y de las organizaciones, 32(3), 173-182. doi: https://doi.org/10.1016/j.rpto.2016.07.001

Guerrero, E. (2002). Modos de afrontamiento de estrés laboral en una muestra de docentes universitarios. Revista interuniversitaria de formación del profesorado, (43), 93-112. Recuperado de https://dialnet.unirioja.es/descarga/articulo/250583.pdf

Gil-Monte, P. (2012). Riesgos psicosociales en el trabajo y salud ocupacional. Revista Peruana de Medicina Experimental y Salud Publica, 29(2), 237-241. Recuperado de http://dx.doi.org/10.17843/rpmesp.2012.292.348

González, N. (2008). Prevalencia del estrés en la satisfacción laboral de los docentes universitarios. REDHECS: Revista electrónica de Humanidades, Educación y Comunicación Social, 3(4), 68-89. Recuperado de https://dialnet.unirioja.es/servlet/articulo?codigo $=2717959$

González, M., \& Landero, R. (2007). Factor structure of the perceived stress scale (PSS) in a sample from Mexico. The Spanish Journal of Psychology, 10(1), 199-206. doi: https://doi.org/10.1017/S1138741600006466

Güilgüiruca, M., Meza, K., Góngora, R., \& Moya, C. (2015). Factores de riesgo psicosocial y estrés percibido en trabajadores de una empresa eléctrica en Chile. Medicina y Seguridad del Trabajo, 61(238), 57-67. doi: https://dx.doi.org/10.4321/S0465$546 \times 2015000100006$

Hernández, R., Fernández, C., \& Baptista, P. (2014). Metodología de la investigación. México: McGram-Hill.

Hiebert, B., \& Farber, I. (1984). Teacher Stress: A Literature Survey with a Few Surprises. Canadian Journal of Education, 9(1), 14-27. doi: https://dx.doi.org/10.2307/1494447

Kline, R. (2005). Principles and practice of structural equation modeling (2 ed.). Nueva York: The Guilford Press.

Kyriacou, C. (2003). Antiestrés para profesores. Barcelona: Octaedro.

Lazarus, R., \& Folkman, S. (1986). Estrés y procesos cognitivos. Barcelona: Martínez Roca.

Luceño, L., Martín, J., Rubio, S., \& Jaén, M. (2008). Psicología y riesgos laborales emergentes, los riesgos psicosociales. EduPsykhé: Revista de psicología y psicopedagogía, 7(2), 111129. Recuperado de https://dialnet.unirioja.es/descarga/articulo/2800121.pdf

Martínez, V. (2003). Estrés y ansiedad en los docentes. Pulso: revista de educación, (26), 9-21. Recuperado de https://revistas.cardenalcisneros.es/index.php/PULSO/article/view/35

Moreno, B. (2011). Factores y riesgos laborales psicosociales: conceptualización, historia y cambios actuales. Medicina y Seguridad del Trabajo, 57(Supl. 1), 4-19. doi: https://dx.doi.org/10.4321/S0465-546X2011000500002

Moreno, B., \& Báez, C. (2010). Factores y riesgos psicosociales, formas, consecuencias, medidas y buenas prácticas. Madrid: Universidad Autónoma de Madrid.

Oramas, A., Rodríguez, R., Almirall, P., Huerta, J., \& Vergara, A. (2003). Algunas causas de estrés psicológico en maestros del Municipio Arroyo Naranjo, Cuba. Revista Cubana de Salud y Trabajo, 4(2), 8-12. Recuperado de http://bvs.sld.cu/revistas/rst/vol4_12_03/rst03103.pdf

Pieró, J. (2001). El estrés laboral: una perspectiva individual y colectiva. Prevención, trabajo y salud: Revista del Instituto Nacional de Seguridad e Higiene en el trabajo, (13), 18-38. Recuperado http://www.insht.es/InshtWeb/Contenidos/Documentacion/TextosOnline/Rev_INSHT/2 001/13/seccionTecTextCompl2.pdf

Peiró, J., \& Rodríguez, I. (2008). Estrés laboral, liderazgo y salud organizacional. Papeles del Psicólogo, 29(1), 68-82. $\quad$ Recuperado de http://www.papelesdelpsicologo.es/resumen?pii=1540

Pérez, P. (2012). El malestar docente. Temas para la educación, 21, 1-6. Recuperado de https://www.feandalucia.ccoo.es/docuipdf.aspx?d=9615\&s 
Raffo, E., Ráez, L., \& Cachay, O. (2013). Riesgos psicosociales. Industrial Data, 16(1), 70-79. doi: https://doi.org/10.15381/idata.v16i1.6420

Restrepo-Ayala, N., Colorado-Vargas, G., \& Cabrera-Arana, G. (2006). Desgaste Emocional en Docentes Oficiales de Medellín, Colombia, 2005. Revista de Salud Pública, 8(1), 63-73. Recuperado de https://www.scielosp.org/article/rsap/2006.v8n1/63-73/

Rodríguez, M. (2009). Factores psicosociales de riesgo laboral: ¿nuevos tiempos, nuevos riesgos? Observatorio laboral revista venezolana, 2(3), 127-141. Recuperado de https://dialnet.unirioja.es/descarga/articulo/2995368.pdf

Rodríguez-Martínez, M., Tovalin-Ahumada, J., Gil-Monte, P., Salvador-Cruz, J., \& AcleTomasini, G. (2018). Trabajo emocional y estresores laborales como predictores de ansiedad y depresión en profesores universitarios mexicanos. Informació Psicològica, (155), 93-107.

Recuperado

de http://www.informaciopsicologica.info/OJSmottif/index.php/leonardo/article/view/852

Rojas, M., \& Rodríguez, J. (2011). Factores de riesgo psicosociales en el profesorado de Enfermería Geriátrica de universidades españolas (2. ${ }^{a}$ parte). Gerokomos, 22(2), 62-71. Recuperado de https://medes.com/publication/67789

Superintendencia de Seguridad Social. (2018). Manual del Método del Cuestionario SUSESO/ISTAS21: Versiones completa y breve. Recuperado de https://www.suseso.cl/606/articles-19640_archivo_05.pdf

Valls-Llobet, C. (2000). El impacto del estrés en la salud de las mujeres. Meridiam, 17, 54-55. Recuperado http://www.juntadeandalucia.es/iam/catalogo/doc/meridiam/meridiam17.pdf 